\title{
Tn554 inserts in methicillin-resistant Staphylococcus aureus from Australia and England: comparison with an American methicillin-resistant group
}

\author{
Sucheta G. Chikramane, ${ }^{1}$ Peter R. Matthews, ${ }^{2}+$ William C. Noble,${ }^{3}$ Peter R. Stewart ${ }^{2}$ \\ and Donald T. DUBIN ${ }^{1 *}$ \\ ${ }^{1}$ Department of Molecular Genetics and Microbiology, University of Medicine and Dentistry of New Jersey-Robert \\ Wood Johnson Medical School, Piscataway, NJ 08854, USA \\ ${ }^{2}$ Division of Biochemistry and Molecular Biology, Faculty of Science, Australian National University, Canberra ACT \\ 2601, Australia \\ ${ }^{3}$ Department of Microbiology, Institute of Dermatology, United Medical and Dental Schools, St Thomas' Hospital, \\ London SE1 7EH, UK
}

(Received 12 October 1990 ; revised 30 January 1991; accepted 13 February 1991)

\begin{abstract}
We have compared methicillin-resistant (Mcr) Staphylococcus aureus isolates from Australia, the UK and the USA with regard to chromosomal inserts of the macrolides-lincosamides-streptogramin B (MLS)-resistance transposon Tn554. The American isolates were known to have a distinctive Tn554 insert, designated insert 6, which was closely associated epidemiologically with the methicillin-resistance phenotype. Southern blots of DNA from Australian and London, $\mathrm{UK} \mathrm{Mcr}$ isolates were hybridized with a range of probes related to Tn554. The isolates had similar or identical Tn554 inserts, and we consider them to be a single group, designated 'Australondon'. Australondon isolates were compared in detail with a deletion mutant, ANS62, that had lost the methicillinresistance determinant $m e c$, plus other resistance determinants resident in the mec region of the chromosome, and with an American Mcr isolate containing Tn554 insert 6. The Australondon isolates had three Tn554 inserts. Sequence analysis with the polymerase chain reaction showed that all of these inserts differed from classical Tn554 in that the $3^{\prime}$-terminal residues of the transposons were reverse complements of the usual GATGTA. One of the Australondon inserts, designated 6B, closely resembled Tn554 insert 6 in the sequence of its left flanking chromosomal DNA. This insert was found to abut the deletion from the mec region which results in strain ANS62. We infer that Tn554 insert 6B is part of the mec region of the chromosome in Australondon isolates, supporting the idea that insert 6 of the American isolates is also part of this chromosomal region.
\end{abstract}

\section{Introduction}

In the past decade, hospitals in New Jersey, USA (Tillotson et al., 1989), eastern Australia (Pavillard et al., 1982; Lyon et al., 1983) and London, UK (Cookson et al., 1986; Cookson \& Phillips, 1988) have suffered outbreaks of infection by methicillin-resistant $\left(\mathrm{Mc}^{\mathrm{r}}\right)$ Staphylococcus

†Present address: The Rockefeller University, York Avenue, New York, NY 10021, USA.

Abbreviations: MLS, macrolides-lincosamides-streptogramin B; PCR, polymerase chain reaction; EMRSA, epidemic methicillinresistant $S$. aureus; $\triangle \mathrm{ANS} 46 / 62$, the DNA deleted from strain ANS46 yielding strain ANS62.

The nucleotide sequence data reported in this paper have been submitted to GenBank and have been assigned the accession number M32312. aureus. The predominant isolates within each location are sufficiently similar to each other to suggest regional spread of a single, or of a few closely related, strain(s). We refer to these sets of isolates as groups. The New Jersey group was identified during a survey for macrolides-lincosamides-streptogramin B (MLS)-resistant staphylococci, and was characterized by Southern blotting DNA from representative isolates with probes related to MLS determinants (Tillotson et al., 1989). This group was MLS due to ermA genes situated in copies of the classical erm $A$-containing chromosomal transposon, $\mathrm{Tn} 554$. $\mathrm{Tn} 554$ is a relatively site-specific transposon that, in experiments with derivatives of the laboratory $S$. aureus strain 8325 , was found to insert into a single (primary) chromosomal attachment site, att554 (Krolewski et al., 1981; Murphy \& Löfdahl, 1984; Murphy, 
1989). However, we have shown that contemporary clinical isolates often have secondary Tn554 inserts, as defined by the presence of inserts that differ substantially from the primary insert in flanking chromosomal DNA restriction maps (Thakker-Varia et al., 1987; Tillotson et al., 1989). We have designated a series of different secondary inserts by the numbers 2 to 8 , in distinction to the primary insert, assigned the number 1 (Tillotson et $a l ., 1989)$. Three of the inserts relevant to the present work are shown in Fig. 1.

The New Jersey $\mathrm{Mc}^{r}$ outbreak group contained, in addition to the primary insert, secondary insert \#6 in chromosomal attachment site att 155, not detected in any $\mathbf{M c}^{\mathrm{s}} \mathrm{S}$. aureus strains. Earlier studies had shown that $\mathbf{M c}^{\mathrm{r}}$ $S$. aureus isolates contain chromosomal DNA, amounting to $30 \mathrm{~kb}$ or more, which is also absent from $\mathrm{Mc}^{\mathrm{s}}$ strains (Beck et al., 1986; Matsuhashi et al., 1986; Matthews et al., 1987). This DNA contains the $\mathbf{M c}^{\mathrm{r}}$ determinant mec and determinants for various other resistances (Skinner et al., 1988; Skurray et al., 1988; Ubukata et al., 1989). It was proposed that Tn554 insert 6 of the New Jersey $\mathrm{Mc}^{\mathrm{r}}$ group might likewise be part of the mec region (Tillotson et al., 1989).

The Australian and London $\mathrm{Mc}^{\mathrm{r}}$ isolates were known to resemble the New Jersey group in having chromosomal MLS determinant(s) (Townsend et al., 1983, 1987; Matthews et al., 1987). To explore further the possibility of a physical relationship between Tn554 inserts and the mec complex of resistance genes, we have characterized the Tn554 elements carried by the Australian and London $\mathrm{Mc}^{\mathrm{r}}$ groups. A variant (ANS62) of the Australian Mcr strain ANS46 that had become $\mathrm{Mc}^{\mathrm{s}}$ because of deletion of part of its mec region was especially informative (Matthews et al., 1987). The Australian and London $\mathrm{Mc}^{\mathrm{r}}$ groups were found to resemble each other and to differ from the New Jersey group by harbouring three Tn554 inserts, all of which had distinctive transposon-chromosomal junction sequences. One of these inserts, designated $6 \mathrm{~B}$, was of special interest. It closely resembled New Jersey Tn554 insert 6 with regard to the left arm of its attachment site and it was indeed found to be in the mec region.

\section{Methods}

Bacterial strains. S. aureus isolates (Table 1) were checked for antibiotic susceptibilities (Barry \& Thornsberry, 1980; Jenssen et al., 1987) just prior to DNA preparation. The plasmid pEM715 carrying a cloned copy of Tn554 was provided by E. Murphy (Public Health Research Institute, New York, USA), in S. aureus strain RN4932 (Thakker-Varia et al., 1987).

DNA methods. Total cellular DNA was isolated after lysing cells with lysozyme, lysostaphin and SDS (Tillotson et al., 1989); proteinase K
Table 1. Bacterial strains

\begin{tabular}{|c|c|c|}
\hline Designation & Origin & Description, and source or reference \\
\hline $\begin{array}{l}\text { N61 } \\
\text { N62 }\end{array}$ & $\begin{array}{l}\text { London, } \\
\text { UK }\end{array}$ & $\begin{array}{l}\text { EMRSA, pT } 181(+) \text { (this work) } \\
\text { EMRSA, pT181(-) (this work) }\end{array}$ \\
\hline ANS46 & \multirow{2}{*}{$\begin{array}{l}\text { Melbourne, } \\
\text { Australia }\end{array}$} & $\begin{array}{l}\text { Prototype Australian epidemic } \mathrm{Mc}^{\mathrm{r}} \\
\text { isolate (Matthews } \text { et al., 1987) }\end{array}$ \\
\hline ANS62 & & $\begin{array}{l}\mathrm{Mc}^{\mathrm{s}} \text { derivative of ANS46 (Matthews } \\
\text { et al., 1987) }\end{array}$ \\
\hline $\mathrm{R} 155^{*}$ & \multirow{2}{*}{$\begin{array}{l}\text { New Jersey, } \\
\text { USA }\end{array}$} & $\begin{array}{l}\text { Prototype New Jersey } \mathrm{Mc}^{\mathrm{r}} \text { isolate, } \\
\text { with a primary Tn554 insert (in } \\
\text { att554), and insert } 6 \text { (in att } 155 \text { ) } \\
\text { (Tillotson et al., 1989) }\end{array}$ \\
\hline WJ137 & & $\begin{array}{l}\text { Prototype New Jersey } \mathrm{Mc}^{\mathrm{s}} \text { isolate, } \\
\text { with a primary Tn554 insert (in } \\
\text { att554), and insert } 2 \text { (in att 137) } \\
\text { (Thakker-Varia et al., 1987) }\end{array}$ \\
\hline
\end{tabular}

- Previously designated R31, this isolate is indistinguishable in our hands from isolate 155 , the strain after which att 155 was named (Tillotson et al., 1989). In the interest of simplicity we renamed it R155.

$\left(200 \mu \mathrm{g} \mathrm{ml}^{-1}\right)$ was substituted for pronase. Southern blot analysis was done as previously described (Thakker-Varia et al., 1987; Tillotson $e t$ al., 1989) except that DNA segment probes were sometimes labelled by random primer extension (Feinberg \& Vogelstein, 1986). Most oligonucleotide probes were designed to have apparent $T_{\mathrm{m}}$ values of 46-48 ${ }^{\circ} \mathrm{C}$, and hybridizations were done at $T_{\mathrm{m}}$ minus $3-5^{\circ} \mathrm{C}$ (Berent $e t$ al., 1986). Filters hybridized with labelled DNA segments were washed twice with $6 \times \mathrm{SSC}(1 \times \mathrm{SSC}$ is $0.15 \mathrm{M}-\mathrm{NaCl}, 15 \mathrm{~mm}$-sodium citrate, $\mathrm{pH} 7 \cdot 0$ ), twice with $1 \times \mathrm{SSC}$ and twice with $0 \cdot 1 \times \mathrm{SSC}$, at room temperature for $20 \mathrm{~min}$. Filters hybridized with labelled oligonucleotides were washed with $90 \mathrm{~mm}$-Tris $/ \mathrm{HCl}(\mathrm{pH} \mathrm{8.0)}$ containing $6 \mathrm{~mm}$ EDTA, $0.9 \mathrm{M}-\mathrm{NaCl}$ and $0.1 \% \mathrm{SDS}$, four times for $10 \mathrm{~min}$ at room temperature, and once for $1 \mathrm{~min}$ at the hybridization temperature (Berent et al., 1985).

Polymerase chain reaction (PCR) amplification of junctional regions was done with Amplitaq (Cetus Corp) according to the supplier's recommended protocol, except that two reactions were run, and primer ratios were adjusted to $20: 1$ or $1: 20$ to facilitate sequencing (Innis $e t$ al., 1988; Mihovilovic \& Lee, 1989). Reactions contained $\approx 1 \mu \mathrm{g}$ DNA and $1.5 \mathrm{mM}-\mathrm{MgCl}_{2} ; 30$ cycles were run in a Perkin-Elmer thermal cycler, at $95^{\circ} \mathrm{C}$ for $1 \mathrm{~min}, 37^{\circ} \mathrm{C}$ for $2 \mathrm{~min}$, and $60^{\circ} \mathrm{C}$ for $2 \mathrm{~min}$.

Sequencing was done by a dideoxynucleotide method (Sanger $e t$ al., 1977) using a T7 DNA polymerase kit (Pharmacia). For the PCR products, we used each low concentration PCR primer alternately as sequencing primer. For sequencing the cloned MAI3 segment of ANS46 DNA, we used as primer oligonucleotide TEM for sequencing out to approximately 150 residues beyond the end of Tn554, and oligonucleotide $\mathrm{T} 53$ as primer for sequencing back on the other strand (see Fig. 1)

\section{Results}

\section{Comparison of Australian and London Mcr isolates}

The results in this and following sections were obtained with a series of probes which are either cloned DNA 


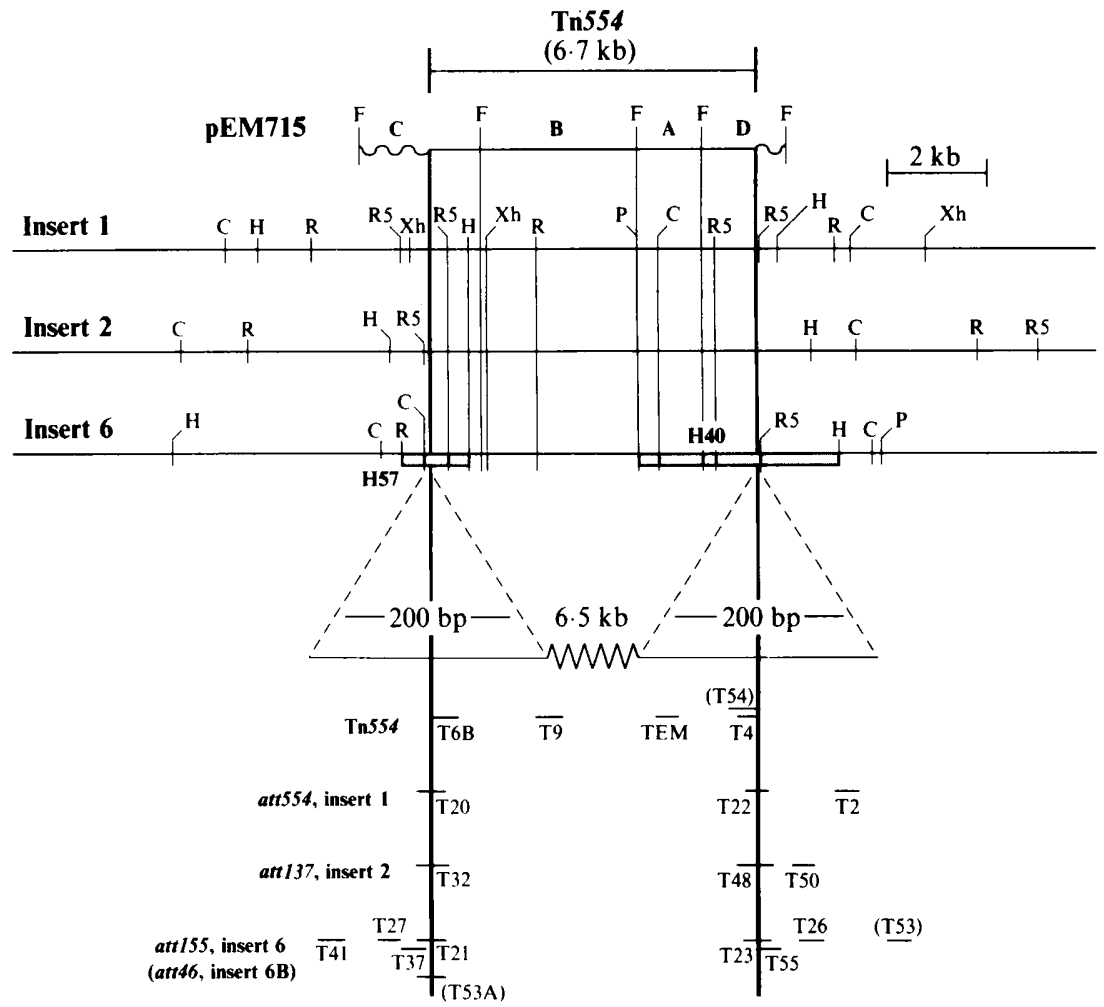

Fig. 1. Physical map of Tn554 inserts found in New Jersey strains, and junction oligonucleotides used to compare variants of these in Australondon strains. At the top we show plasmid pEM715 (Thakker-Varia et al., 1987), used as source of Tn554 segment probes, with Fnu4HI (F) sites bounding the segments used. The wavy lines represent sequence from the pT181 vector (Khan \& Novick, 1983). Below, we diagram the primary insert (\#1) and secondary inserts (\# 2, \#6) found previously (Murphy \& Löfdahl, 1984; Thakker-Varia et al., 1987; Tillotson et al., 1989). Junction chromosomal sites for XhoI (Xh), EcoRV (R5), Pst I (P), EcoRI (R), HindIII (H) and ClaI (C) that are within $7 \mathrm{~kb}$ of transposon boundaries, as well as relevant sites (Murphy et al., 1985) within the transposon, are shown. H40 and H57 designate cloned junctional fragments from insert 6 (Tillotson et al., 1989). The bold vertical lines indicate the boundaries of $\mathrm{Tn} 554$. We follow the convention of E. Murphy and colleagues (Murphy \& Löfdahl, 1984; Murphy et al., 1985; Murphy, 1989) here and elsewhere with regard to orientation of the transposon; the major coding strand is presented $5^{\prime}$ to $3^{\prime}$, left to right. An expanded map of insert boundaries is shown in the lower half of the figure. Oligonucleotides which were used in the comparative analysis of the Australondon and New Jersey strains are shown, with Australondon-specific ones in parentheses. Precise sizes and locations of oligonucleotide sequences are given in Table 2.

fragments of Tn554 inserts (Fig. 1) or oligonucleotides corresponding to Tn554 or Tn554 attachment sites (Fig. 1 and Table 2).

Eight recent $\mathrm{Mc}^{\mathrm{r}} \mathrm{MLS}$ isolates from London Hospitals were compared with the Australian $\mathrm{Mc}^{\mathrm{r}}$ strain ANS46 and the New Jersey Mcr strain R155. Total cellular DNA was digested with EcoRI, HindIII, or HindIII plus PstI. Southern blots were probed with cloned Tn554 primary insert fragments $\mathrm{C}$ and D from plasmid pEM715 (which contain also some sequence from the vector $\mathrm{pT} 181$ ), and with junction fragment $\mathrm{H} 40$ of insert 6 (Fig. 1). Five of the London isolates contained Tn554, and with the exception of one feature (the absence of integrated plasmid pT181, as described below) were indistinguishable from each other and from the Australian $\mathrm{Mc}^{\mathrm{r}}$ isolate ANS46 (data not shown). These five London isolates had antibiotic susceptibility patterns, including the 'inducible-constitutive' MLS phenotype, characteristic of eastern Australian isolates of $\mathrm{Mc}^{\mathrm{r}}$ S. aureus (Townsend et al., 1983) and of a group of epidemic $\mathrm{Mc}^{\mathrm{r}}$ isolates from London (termed EMRSA, following Cookson \& Phillips, 1988) (Townsend et al., 1987). We consider these five London isolates to be EMRSA. These results confirm that the EMRSA and the Australian epidemic strains are closely related (Townsend et al., 1987), and we shall refer to them as the Australondon group.

The feature of the initial Southern blot results that distinguished two of the five London EMRSA isolates from ANS46 was the absence of a chromosomal copy of the pT181-like plasmid (Khan \& Novick, 1983) in the mec region (Gillespie et al., 1986; Skinner et al., 1988); these two EMRSA isolates failed to hybridize with probes containing pT181 sequences. However, they were 
resistant to tetracycline and minocycline (albeit slightly less so than the other three EMRSA), probably due to the presence of tet $M$, located elsewhere on the chromosome (Lyon \& Skurray, 1987; Cookson \& Phillips, 1988). Results for ANS62 were the same as those for the EMRSA isolates lacking pT181; i.e. tet $M$ appears to remain and is therefore, as expected, not in the DNA segment (' $\triangle \mathrm{ANS} 46 / 62$ ') deleted from ANS46 to give strain ANS62.

\section{Southern hybridization analysis of Tn554 inserts of Australondon $\mathrm{Mc}^{\mathrm{r}}$ isolates: comparison with a $\mathrm{Mc}^{\mathrm{s}}$ variant and with New Jersey isolates}

The six strains listed in Table 1 were chosen for more detailed analysis. Chromosomal DNA samples were digested with six restriction endonucleases, singly and in pairs, as indicated in the legend to Fig. 1.

Southern blots were probed sequentially with Tn554related oligonucleotides (Fig. 1 and Table 2), and with fragments from Tn554 (Fig. 1). We show representative Southern hybridization patterns in Figs 2 and 3. These illustrate the complexity of hybridization patterns obtained from Australondon DNA when relatively nonspecific probes are used (Fig. $2 a$ ), and the clearer picture which emerges when more specific probes are used (Figs $2 b, c$ and 3 ). The complexity of the patterns relates to the presence of three Tn554 inserts in different attachment sites in the chromosome: \# 1 is indistinguishable from the primary insert in att554; \#2B resembles insert 2 (Thakker-Varia et al., 1987); and \#6B (6B' in ANS62, as described below) resembles insert 6 (Tillotson et al., 1989).

\section{Oligonucleotide probe design}

To detect junction restriction fragments, filters were hybridized with oligonucleotides corresponding to left and right regions of Tn554 (see Fig. 1 and Table 2). Oligonucleotide T6B, corresponding to the 5 '-terminal 21 residues of the transposon, was used to detect left junction fragments of the Australondon inserts. Oligonucleotide TEM was used to detect right junction fragments, since oligonucleotide T4 (which corresponds more closely to $\mathrm{T} 6 \mathrm{~B}$ as a right terminal transposon probe) did not recognize DNA from Australondon isolates.

Oligonucleotides of higher specificity were designed to facilitate assignment of hybridizing fragments to a particular Tn554 insert class. We initially used oligonucleotides spanning transposon-chromosomal junctions as determined for American strains (Fig. 1): T20 and $\mathrm{T} 22$ for the primary insert, $\mathrm{T} 21$ and $\mathrm{T} 23$ for insert 6 , and $\mathrm{T} 32$ and $\mathrm{T} 48$ for insert 2 . As expected from the
Table 2. Oligonucleotide probes: hybridization with Australondon DNA

\begin{tabular}{|c|c|c|c|c|}
\hline \multirow[b]{2}{*}{ Oligonucleotides* } & \multicolumn{4}{|c|}{ Australondon inserts $\dagger$} \\
\hline & 1 & $2 \mathrm{~B}$ & $6 \mathrm{~B}$ & $6 \mathrm{~B}^{\prime}$ \\
\hline \multicolumn{5}{|l|}{$\operatorname{Tn} 554$} \\
\hline T6B +21 to +1 (L end) & + & + & + & + \\
\hline $\mathrm{T} 9+129$ to +112 (L penultimate) & + & + & + & + \\
\hline TEM -82 to -65 ( $R$ penultimate) & + & + & + & + \\
\hline $\mathrm{T} 4-15$ to $-1(\mathrm{R}$ end $)$ & - & - & - & - \\
\hline T54 -19 to -1 (R end) $\ddagger$ & + & + & + & + \\
\hline \multicolumn{5}{|l|}{ att 554, insert 1} \\
\hline $\mathrm{T} 20+4$ to -13 (L junction) & + & - & - & - \\
\hline $\mathrm{T} 22-9$ to +7 ( $\mathrm{R}$ junction) & - & - & - & - \\
\hline $\mathrm{T} 2+79$ to $+61(\mathrm{R}$ arm $)$ & + & - & - & - \\
\hline \multicolumn{5}{|l|}{ att 137, insert 2} \\
\hline $\mathrm{T} 32-11$ to +6 (L junction) & - & + & - & - \\
\hline $\mathrm{T} 48-13$ to +6 ( $\mathrm{R}$ junction) & - & - & - & - \\
\hline $\mathrm{T} 50+29$ to $+44(\mathrm{R}$ arm $)$ & - & + & - & - \\
\hline \multicolumn{5}{|l|}{ att 155 , insert 6} \\
\hline $\mathrm{T} 41-65$ to $-85(\mathrm{~L}$ arm $)$ & - & - & + & + \\
\hline $\mathrm{T} 27-40$ to -24 (L arm) & - & - & + & + \\
\hline T37 -26 to $-10(\mathrm{~L}$ arm $)$ & - & - & + & + \\
\hline $\mathrm{T} 21+5$ to -12 (L junction) & - & - & - & - \\
\hline $\mathrm{T} 23-9$ to +8 ( $\mathrm{R}$ junction) & - & - & - & - \\
\hline $\mathrm{T} 55+1$ to $+15(\mathrm{R}$ arm $)$ & - & - & - & - \\
\hline $\mathrm{T} 26+52$ to $+33(\mathrm{R}$ arm $)$ & - & - & - & - \\
\hline \multicolumn{5}{|l|}{ att 46 , insert 6B } \\
\hline $\mathrm{T} 53 \mathrm{~A}-11$ to +6 (L junction) & - & - & + & + \\
\hline $\mathrm{T} 53+111$ to $+94(\mathrm{R}$ arm $)$ & - & - & + & - \\
\hline
\end{tabular}

* Residue numbers indicate nucleotide positions to the left (negative numbers) or right (positive numbers) of the relevant transposonchromosome junction. The left column of numbers specifies the $5^{\prime}$, and the right column the $3^{\prime}$, ends of the oligonucleotides. The numbering convention is illustrated in Figs 4 and 5 for several oligonucleotides. Sequences may be found as follows: Tn554, Murphy et al. (1985); att554 and insert 1, Murphy \& Löfdahl (1984); att137 and insert 2, Dubin et al. (1990); att155 and insert 6, Tillotson et al. (1989); att46 and insert 6B, present work, Figs 4 and 5.

† Strong hybridization $(+)$ indicates no mismatches, or at most one terminal mismatch, between target and probe under the hybridization conditions used.

$\ddagger$ Right transposon end except with reverse complement of the usual GATGTA extreme terminal hexanucleotide.

results for right transposon-terminal oligonucleotide $\mathrm{T} 4$, the right junction oligonucleotides failed to recognize any of the Australondon inserts. Unexpectedly, the oligonucleotide corresponding to the left junction of insert 6, T21, failed to recognize Australondon insert 6B. However, using oligonucleotides TEM and T2 (Fig. 1), a 161-nucleotide product spanning the right junction of the primary insert of one of the London strains (N56) was generated by PCR. Similarly, we used Tn554 oligonucleotide T9 and left att 155 oligonucleotide T27 (Fig. 1) to generate a 169-nucleotide product spanning the left junction of ANS46 insert 6B. Both PCR products were then sequenced.

The extent to which the Australondon junction sequences differ from their American homologues is 
(a) Tn554, Segment B

$-6 \mathrm{~L}$

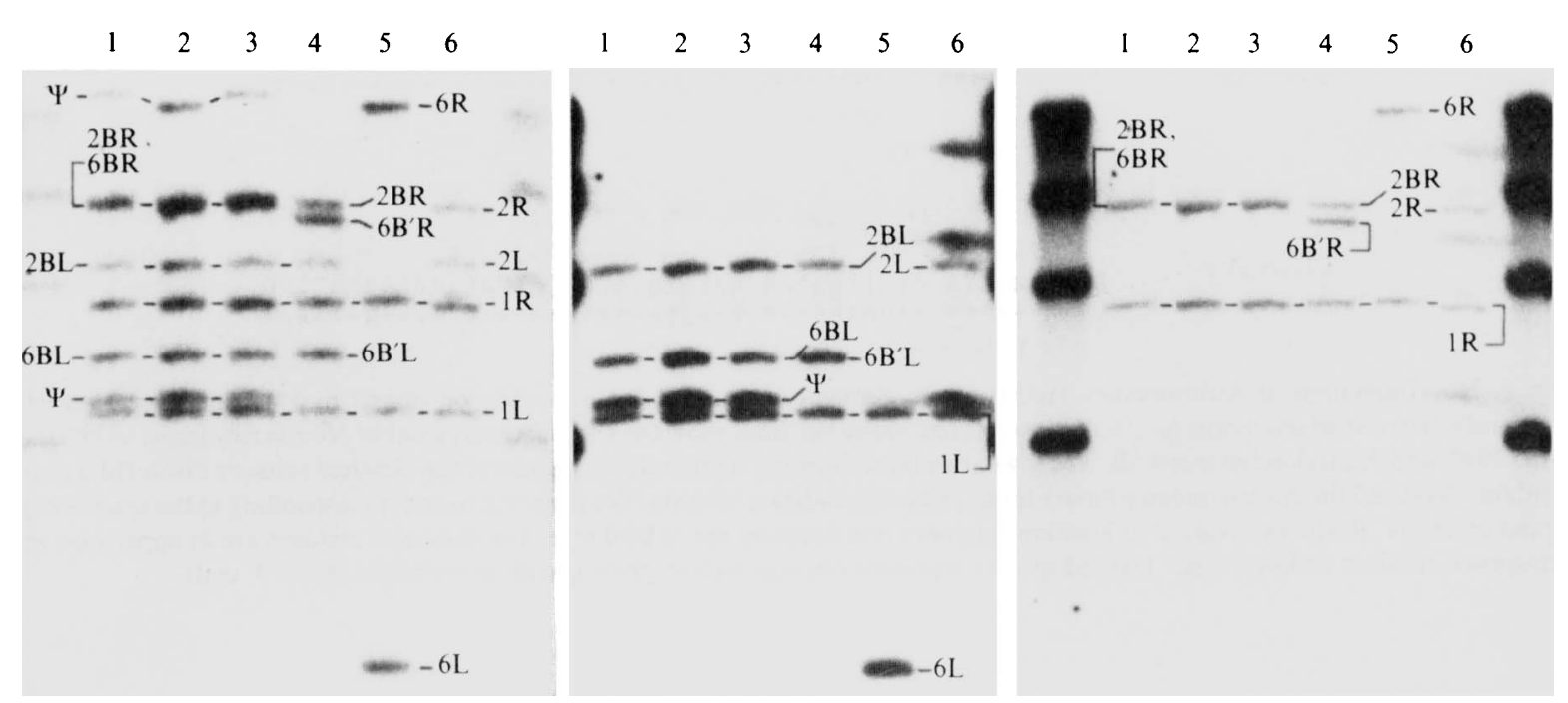

(b) T6B

(c) TEM

Fig. 2. EcoRI digests of chromosomal DNA. Here and in Fig. 3 we show blots hybridized with probes of least specificity on the left, so that the left-most patterns give an overall picture, and the other patterns identify more precisely certain of the bands. Patterns represent filters hybridized sequentially with different probes, as indicated for $(a),(b)$ and $(c)$. The end lanes show bands from HindIII digests of bacteriophage $\lambda$ used as size standards: $23 \mathrm{~kb}, 9.4 \mathrm{~kb}, 6.6 \mathrm{~kb}, 4.4 \mathrm{~kb}$, and (for Fig. 3) $2.3 \mathrm{~kb}$ and $2.0 \mathrm{~kb}$. The other lanes (1-6) show, in order, digests of DNA from London isolates N61 and N62; Australian strains ANS46 and ANS62; and New Jersey isolates R155 and WJ137 (described in Table 1). Bands are identified as to source. For Tn554 inserts we indicate insert number and whether left (L) or right (R) junction. Bands from ' $\Psi$ Tn554 (see text) are designated ' $\Psi$ '. The streaks in lane 6 lacking identifying labels are artifacts from the adjacent marker lane.

(a) TEM

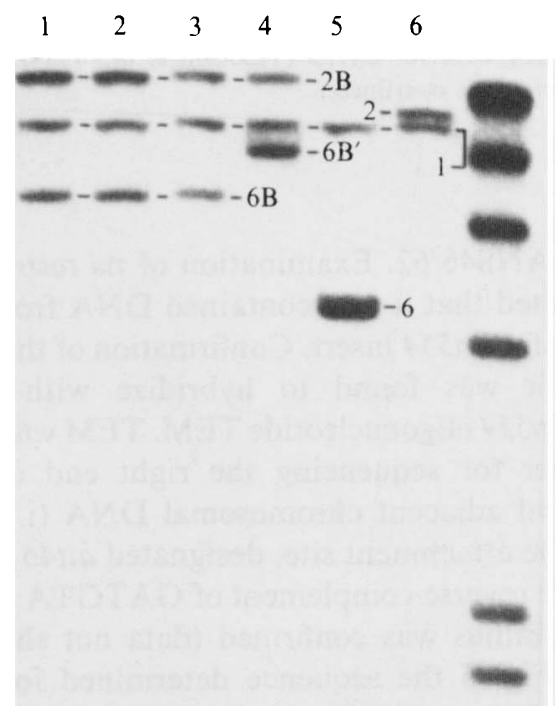

(b) T54

$\begin{array}{llllll}1 & 2 & 3 & 4 & 5 & 6\end{array}$

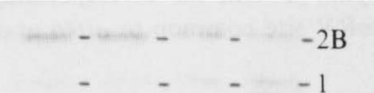

$-\frac{-1}{-6 B}$

$-6 \mathrm{~B}$ (c) $\mathrm{T} 53$

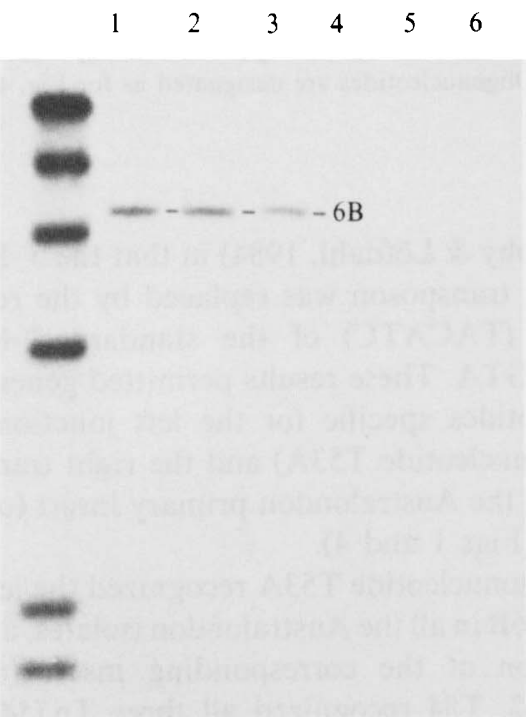

Fig. 3. Pst I digests. The plan is as for Fig. 2. Bands are identified as in that Figure, all being from right junctions.

shown in Fig. 4. At the left junction of insert 6B, the seven residues of the chromosome adjacent to $\operatorname{Tn} 554$ were found to differ from those of insert 6 in two positions, one of which converts the otherwise conserved GATGTA core of the attachment site (Murphy, 1989) to
GATATA. Interestingly, this is one of few core variants found to retain full activity in experiments with in vitro core mutants (E. Murphy, personal communication). The right junction of the primary insert of strain N56 was found to differ from standard versions of this junction 


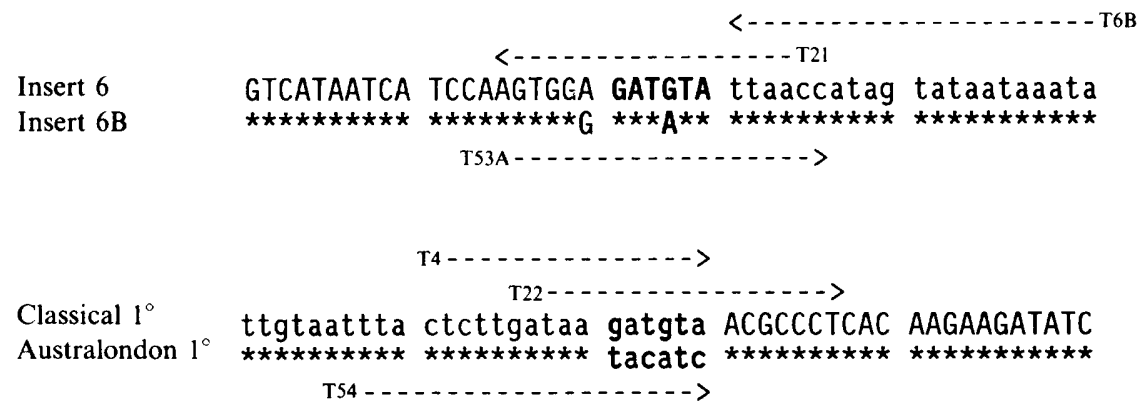

Fig. 4. Novel junctions of Australondon Tn554 inserts. Sequences obtained for Australondon inserts by PCR are compared with previously determined sequences for similar inserts. The upper two lines show the left junction regions of New Jersey insert 6 (Tillotson et al., 1989) and Australondon insert 6B. The lower two lines show the right junction regions of the classical primary insert (Murphy \& Löfdahl, 1984) and the Australondon primary insert; asterisks indicate identity. We show the strand corresponding to the main coding strand of $\mathrm{Tn} 554$. Residues involved in junction fragment translocation are in bold type; chromosomal residues are in upper case and transposon residues in lower case. Dashed arrows represent oligonucleotide probes, with arrowheads at the $3^{\prime}$ ends.

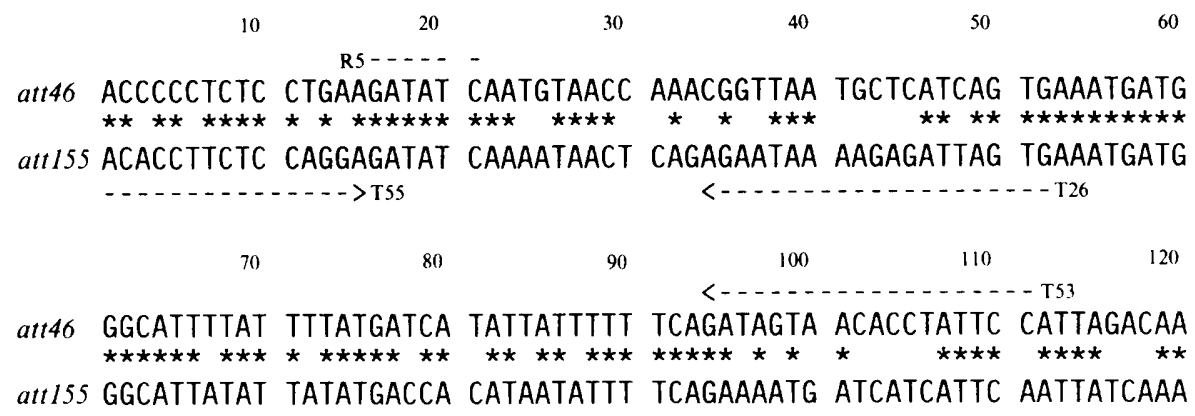

Fig. 5. Sequence of right adjacent chromosomal DNA of insert 6B (right arm of att46) of ANS46. We show the sequence beginning at the first nucleotide beyond the transposon, and compare it with the corresponding sequence for att155 (Tillotson et al., 1989). Oligonucleotides are designated as for Fig. 4. The EcoRV site common to att46 and att 155 is overlined.

(Murphy \& Löfdahl, 1984) in that the 3'-hexanucleotide of the transposon was replaced by the reverse complement (TACATC) of the standard 3'-hexanucleotide GATGTA. These results permitted generation of oligonucleotides specific for the left junction of insert $6 \mathrm{~B}$ (oligonucleotide T53A) and the right transposon terminus of the Australondon primary insert (oligonucleotide T54) (Figs 1 and 4).

Oligonucleotide T53A recognized the left junctions of insert $6 \mathrm{~B}$ in all the Australondon isolates, and also the left junction of the corresponding insert $\left(6 \mathrm{~B}^{\prime}\right)$ of strain ANS62. T54 recognized all three Tn554 inserts of all Australondon isolates, and of strain ANS62; thus the transposons of all these inserts have reverse complements of the standard GATGTA at their 3'-termini.

A third Australondon-specific oligonucleotide was based on sequence data for the right chromosomal arm of insert 6B, obtained from cloned fragment MA13 (Matthews et al., 1990). This fragment is the leftmost of those cloned from the mec region of ANS46 that contain
DNA from $\triangle \mathrm{ANS} 46 / 62$. Examination of its restriction pattern suggested that it also contained DNA from the right portion of a Tn554 insert. Confirmation of this was given when it was found to hybridize with right penultimate Tn 554 oligonucleotide TEM. TEM was then used as primer for sequencing the right end of the transposon, and adjacent chromosomal DNA (i.e. the right arm of the attachment site, designated att46). The presence of the reverse complement of GATGTA at the transposon terminus was confirmed (data not shown). We show in Fig. 5 the sequence determined for 120 residues of att 46 beyond this terminus, and compare it with the corresponding region of the attachment site (att155) of New Jersey insert 6. Homology between the two stretches was only $70 \%$, which permitted design of an oligonucleotide probe (T53) specific for the right chromosomal arm of insert 6B. This oligonucleotide recognized insert $6 \mathrm{~B}$ in all the Australondon isolates examined; it failed to recognize any other inserts, including insert $6 \mathrm{~B}^{\prime}$ of ANS62. 


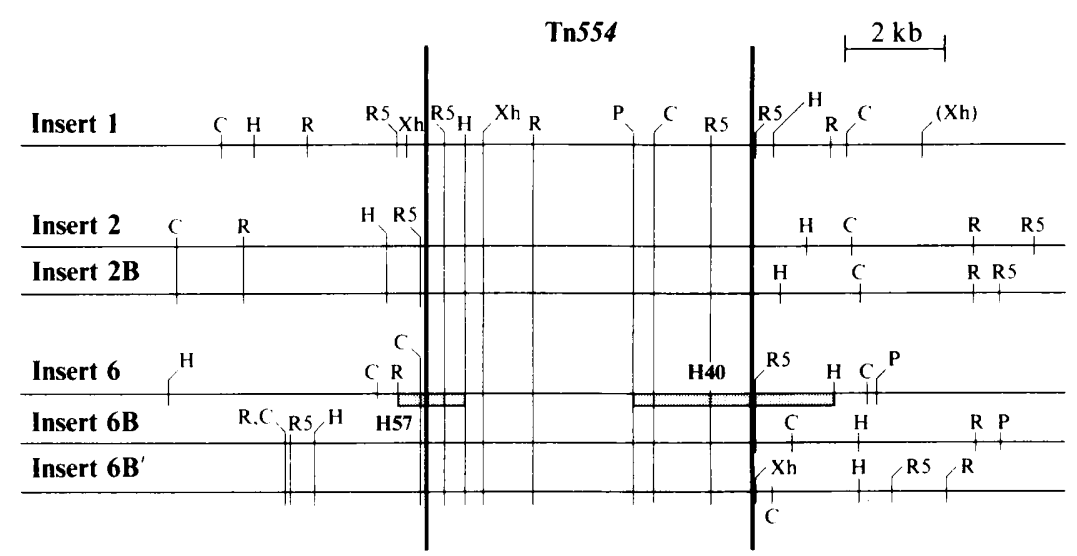

Fig. 6. Physical maps of Tn554 inserts of Australondon isolates. Below the maps of inserts 2 and 6 we show comparable maps for Australondon inserts $2 \mathrm{~B}$ and $6 \mathrm{~B}$, and insert $6 \mathrm{~B}^{\prime}$ of the Australondon deletant strain, ANS62. Format and abbreviations are as for Fig. 1.

\section{Hybridization data}

Fig. 2 shows a Southern blot of EcoRI digests of DNA from the six representative isolates used in this study. In Fig. 2(a), DNA was probed with internal Tn554 fragment B. This fragment spans the single EcoRI site of the transposon (Fig. 1), thus generating labelled left and right junction fragments and yielding a complex hybridization pattern. Seven well-defined bands are seen in the blots of the Australondon isolates (lanes 1-3). These were sorted out by hybridization with more specific oligonucleotides as exemplified by Fig. $2(b, c)$, and by comparison with the simpler patterns of strains WJ137 and R155, which contain the primary insert plus, respectively, inserts 2 or 6 (Thakker-Varia et al., 1987; Tillotson et al., 1989). Five of the Australondon EcoRI bands of Fig. 2(a) come from three Tn554 inserts in each of these strains, with two junction fragments (2BR, 6BR) comigrating.

Two EcoRI bands (designated $\Psi$ ) arise in part from a Tn554-related element (designated $\Psi T n 554$, for pseudotransposon) that is located in the mec region of the Australondon strains. This element hybridized with Tn554 fragment B (Fig. 2a) and with oligonucleotide T6B (Fig. 2b). We shall describe $\Psi \operatorname{Tn} 554$ in detail elsewhere, but note here that the largest of the EcoRI fragments of Fig. 2(a) (lanes 1 and 3) includes the pT181 insert as well as a portion of $\Psi \operatorname{Tn} 554$. The slightly faster migration of the N62 version of this band (lane 2), compared to N61 and ANS46 (lanes 1 and 3), reflects the absence of pT181 from N62.

Hybridization patterns for ANS62, the deletion mutant of ANS46, are shown in Fig. 2, lanes 4. These lanes lack the bands arising from $\Psi T$ Tn554 (Fig. $2 a, b$ ). Further, the ANS62 patterns include a band, designated $6 \mathrm{~B}^{\prime} \mathrm{R}$, not present in wild-type Australondon isolates, and lack $E c o$ RI fragment $6 \mathrm{BR}$, as reflected in the $E c o$ RI digests by the relative decrease in intensity of the Australondon composite 2BR/6BR bands (Fig. 2a,c). This is seen more clearly in Fig. 3, which shows right $P$ st I fragments. These are well separated, and the size difference between right junctional fragments from insert $6 \mathrm{~B}$ versus insert $6 \mathrm{~B}^{\prime}$ (Fig. $3 a, b$ ) is evident. The specificity of right transposon-terminal oligonucleotide T54 (see Fig. 4) for Australondon isolates is shown in Fig. $3(b)$. As noted above, T54, which contains no flanking chromosomal nucleotides, hybridizes to all the Tn554 inserts of the Australondon group. It hybridizes also to insert $6 \mathrm{~B}^{\prime}$ of ANS62. [For unknown reasons, bands arising from the right junction of insert $6 \mathrm{~B}^{\prime}$ were often disproportionately intense (Fig. 3a,b).] Fig. 3(c) shows the specific hybridization of right junctional oligonucleotide T53 to insert 6B of the wild-type Australondon isolates, but not to insert $6 \mathrm{~B}^{\prime}$. These results taken together show that the right junctional fragment of insert 6B in ANS46 spans a boundary of the segment of DNA whose deletion resulted in ANS62.

A summary of oligonucleotide hybridization experiments, including those for which hybridization data are not shown, is given in Table 2 . These results, together with results with cloned $\mathrm{Tn} 554$ fragments, were used to generate restriction maps for all insertion sites represented in the Australondon and New Jersey isolates (Fig. 6).

The maps of the primary insert of the Australondon isolates were essentially the same as those of the classical primary insert 1 . Australondon insert $2 \mathrm{~B}$, which resembles insert 2 by oligonucleotide hybridization (Table 2), had a left junction restriction map that was indistinguishable from that of insert 2. The map of the right junction showed minor differences.

The third Australondon Tn554 insert was designated $6 \mathrm{~B}$ on the basis of the high similarity of the sequence of its left flanking DNA to that of insert 6 out to residue 85 (Fig. 4 and Table 2). Other hybridization data (not 
shown) indicated that the left flanking chromosomal DNA sequences of these two inserts were highly similar for up to $0.5 \mathrm{~kb}$ from the transposon, and then diverged (Fig. 6). In contrast, the right flanking chromosomal DNA of these two inserts diverged significantly beginning a few nucleotides from the transposon (Figs 5 and 6).

The restriction maps of the left flanking DNA of inserts $6 \mathrm{~B}$ and $6 \mathrm{~B}^{\prime}$ were the same, whereas those of corresponding right flanking DNA were completely different from each other, apart from an apparently fortuitous concordance of HindIII sites (Fig. 6, lowest two lines).

\section{Discussion}

\section{Multiplicity of Tn554 inserts in the Australondon group}

The occurrence of three Tn554 inserts in the Australondon isolates is not unprecedented (Tillotson et al., 1989). Whether the accretion of Tn554 inserts confers an advantage on a strain is unknown. However, it seems likely that the three copies of Tn554 confer upon Australondon strains the higher basal (uninduced) level of MLS (compared to classical inducible strains) characteristic of this group (Townsend et al., 1983).

\section{Ancestry of Australondon Tn554}

The different terminal 3'-hexanucleotide (TACATC) of the three Tn554 elements in the Australondon group, compared with those of isolates characterized earlier, can be explained by junction fragment translocation that occurs during Tn554 transposition (Murphy, 1989; Dubin et al., 1990). The 3'-terminal six or seven nucleotides of a transposon in a particular site constitute a 'copy' of the six or seven chromosomal nucleotides 5 ' to the parental transposon (i.e. the core of the parental attachment site). Tn554 inserts preferentially in a single orientation, designated $(+)$, in its primary chromosomal site, and transposition occurs preferentially into this site, with insertion occurring just $3^{\prime}$ of the attachment site core, GATGTA. This, coupled with junction translocation (not conventional target duplication), explains the preponderance of inserts with 'copies' of the GATGTA core at the 3 -ends of the transposons, at least in laboratory strains (Murphy \& Löfdahl, 1984; Murphy, 1989) derived from the original Wisconsin isolate (Weisblum \& Demohn, 1969). On the other hand, Tn554 readily inserts in ( - ) orientation in plasmids (Murphy \& Löfdahl, 1984) and can do so, albeit rarely, in the chromosome of laboratory strains (Murphy \& Löfdahl, 1984; Murphy, 1989). Such (-) insertions occur immedi- ately $5^{\prime}$ of the attachment site core. Thus, for a (-) insert in a primary site, this core is seen as the reverse complement, TACATC, of the usual GATGTA. The inserts of the Australondon group must have arisen by transposition from one or more $(-)$ inserts in a primary, or primary-like, site. The nature of the transposon donor is open to speculation, but interesting candidates are Western Australian strains with Tn554 inserts in conjugative plasmids (Townsend et al., 1986).

\section{Tn554 inserts $6 B$ and 6 , and their physical relationship to mec}

Perhaps our most important findings pertain to physical linkage between particular Tn554 inserts and mec. The present findings show that this linkage exists for insert $6 \mathrm{~B}$ of the Australondon group. There is good evidence that the only difference between Australian isolate ANS46 and ANS62 is the absence from ANS62 of about $40 \mathrm{~kb}$ of contiguous DNA, $\triangle \mathrm{ANS} 46 / 62$, containing mec and resistance determinants for tetracycline, cadmium and mercury (Matthews et al., 1987; Inglis et al., 1988; Skinner et al., 1988). The difference between the right junction fragments of ANS46 insert 6B and ANS62 insert $6 \mathrm{~B}^{\prime}$ shows that insert $6 \mathrm{~B}$ is at or near a boundary of $\triangle \mathrm{ANS46/62}$. We have established that this is the left boundary (as conventionally drawn; see Matthews et al., 1987) by demonstrating that a portion of insert $6 \mathrm{~B}$ is included in clone MA13 from ANS46; this clone, in turn, had been shown to contain DNA from the left portion of $\triangle \mathrm{ANS} 46 / 62$ (Matthews et al., 1990).

An $E c o$ RV site 16-21 residues $3^{\prime}$ to the transposon of insert 6B (Fig. 5) is missing from insert 6 $\mathrm{B}^{\prime}$ (Fig. 6). In addition, the transposon-terminal oligonucleotide T54 hybridized with insert $6 \mathrm{~B}^{\prime}$ as well as with insert $6 \mathrm{~B}$ (Table 2 ), indicating that the left deletion boundary in ANS46 is within 21 residues of the transposon. It is quite possible that the two boundaries correspond precisely, which of course would directly implicate the transposon in the deletion event.

The similarity between the right flanking DNA of inserts 6 and $6 \mathrm{~B}$ (i.e. the right arms of att 155 and att46) is low compared to the striking similarity between the respective left arms. This implies common ancestry for the left, but not the right, arms, and suggests that during the divergence of the Australondon and New Jersey lineages the arms of an ancestral attachment site (att155like or att46-like, whichever is older) must have become separated.

Several lines of evidence suggest that insert 6 is in the mec region of the New Jersey lineage: $(a)$ the epidemiological association between the insert and $\mathrm{Mc}^{\mathrm{r}}$ (Tillotson et al., 1989); (b) the similarity between the left flanking DNA of inserts 6 and $6 \mathrm{~B}$; and $(c)$ the fact that 
insert 6B is in the mec region. Further work is in progress better to define the structure of this variant of mec region DNA and its relationship with the Australondon version.

This work was supported in part by Grant 20.88 from the Foundation of the University of Medicine and Dentistry of New Jersey (D.T.D.), and by the National Health and Medical Research Council of Australia (P.R.S.). We thank Lotus Moon-McDermott, Barbara Inglis and Sheila Mazar for their expert technical assistance.

\section{References}

Barry, A. L. \& Thornsberry, C. (1980). Susceptibility testing: diffusion test procedures. In Manual of Clinical Microbiology, 3rd edn, pp. 463-474. Edited by E. A. Lennette. Washington, DC: American Society for Microbiology.

BeCK, W. D., BERGER-BACHI, B. \& KAYSER, F. H. (1986). Additional DNA in methicillin-resistant Staphylococcus aureus and molecular cloning of mec-specific DNA. Journal of Bacteriology 165, 373-378.

Berent, S. L., Mahmoudi, M., Torczynski, R. M., BragG, P. W. \& Bollon, A. P. (1985). Comparison of oligonucleotide and long DNA fragments as probes in DNA and RNA dot, Southern, Northern, colony and plaque hybridization. BioTechniques 3, 208-220.

Cookson, B. D. \& Phillips, I. (1988). Epidemic methicillin-resistant Staphylococcus aureus. Journal of Antimicrobial Chemotherapy 21 (suppl. C), 57-65.

Cookson, B. D., Talsania, H., Naidoo, A. \& Phillips, I. (1986). Strategies for typing and properties of epidemic methicillin-resistant Staphylococcus aureus. European Journal of Clinical Microbiology 5 , 702-709.

Dubin, D. T., Tillotson, L. E., Huwyler, L. \& Murphy, E. (1990). Erm methylases in staphylococci and their mode of spread. In Nucleic Acid Methylation, pp. 125-137. Edited by G. Clawson, D. Willis, A. Weissbach \& P. Jones. New York: Alan R. Liss.

Feinberg, A. P. \& Vogelstein, B. (1986). A technique for radiolabeling DNA restriction endonuclease fragments to high specific activity. Analytical Biochemistry 132, 6-13.

Gillespie, M. T., May, J. W. \& Skurray, R. A. (1986). Detection of an integrated tetracycline resistance plasmid in the chromosome of methicillin resistant Staphylococcus aureus. Journal of General Microbiology 132, 1723-1728.

Inglis, B., Matthews, P. R. \& Stewart, P. R. (1988). The expression in Staphylococcus aureus of cloned DNA encoding methicillin resistance. Journal of General Microbiology 134, 1465-1469.

InNis, M. A., Myambo, K. B., Gelfand, D. H. \& Brow, M. D. (1988). DNA sequencing with Thermus aquaticus DNA polymerase and direct sequencing of polymerase chain reaction amplified DNA. Proceedings of the National Academy of Sciences of the United States of America 88, 9436-9440.

Jenssen, W. D., Thakker-Varia, S., Dubin, D. T. \& Weinstein, M. P. (1987). Prevalence of macrolides-lincosamides-streptogramin B resistance and erm gene classes among clinical strains of staphylococci and streptococci. Antimicrobial Agents and Chemotherapy 31, 883-888.

KhaN, S. A. \& Novick, R. P. (1983). Complete nucleotide sequence of pT181, a tetracycline-resistance plasmid from Staphylococcus aureus. Plasmid 10, 251-256.

Krolewski, J. J., Murphy, E., Novick, R. P. \& RuSh, M. D. (1981). Site-specificity of the chromosomal insertion of Staphylococcus aureus transposon Tn554. Journal of Molecular Biology 152, 19-33.

Lyon, B. R., MaY, J. W. \& Skurray, R. A. (1983). Analysis of plasmids in nosocomial strains of multiple-antibiotic-resistant Staphylococcus aureus. Antimicrobial Agents and Chemotherapy 23, 817-826.

Lyon, B. R. \& SKURRAY, R. A. (1987). Antimicrobial resistance of Staphylococcus aureus: genetic basis. Microbiological Reviews 51, 88134.
Matsuhashi, M., Song, M. D., Ishimo, F., WaChi, M., Doi, M., Inoue, M., Ubukata, K., Yamashita, N. \& Konno, M. (1986). Molecular cloning of the gene of a penicillin-binding protein supposed to cause high resistance to beta-lactam antibiotics in Staphylococcus aureus. Journal of Bacteriology 167, 975-980.

Matthews, P. R., Reed, K. C. \& Stewart, P. R. (1987). The cloning of chromosomal DNA associated with methicillin and other resistances in Staphylococcus aureus. Journal of General Microbiology 133, 1919-1929.

Matthews, P. R., Inglis, B. \& Stewart, P. R. (1990). Clustering of resistance genes in the mec region of the chromosome of Staphylococcus aureus. In Molecular Biology of the Staphylococci, pp. 59-83. Edited by R. Novick. New York: VCH Publishing.

Minovilovic, M. \& LeE, J. E. (1989). An efficient method for sequencing PCR amplified DNA. BioTechniques 7, 14-16.

MURPHY, E. (1989). Transposable elements in Gram-positive bacteria. In Mobile DNA, pp. 269-288. Edited by D. Berg \& M. Howe. Washington, DC: American Society for Microbiology.

MURPHY, E. \& LöFDAHL, S. (1984). Transposition of Tn554 does not generate a target duplication. Nature, London 300, 292-294.

Murphy, E., HuWyler, L. \& Bastos, M. C. (1985). Transposon Tn554: complete nucleotide sequence and isolation of transpositiondefective and antibiotic-sensitive mutants. EMBO Journal 4, 33573365.

Pavillard, R., Harvey, K., Douglas, D., Hewstone, A., Andrew, J., Collopy, B., Asche, V., Carson, P., Davidson, A., Gilbert, G., SPICER, J. \& Tosolini, F. (1982). Epidemic of hospital-acquired infection due to methicillin-resistant Staphylococcus aureus in major Victorian hospitals. Medical Journal of Australia 1, 451-454.

Sanger, F., Nicklen, S. \& Coulson, A. R. (1977). DNA sequencing with chain-terminating inhibitors. Proceedings of the National Academy of Sciences of the United States of America 74, 5463-5467.

Skinner, S., Inglis, B., Matthews, P. R. \& Stewart, P. R. (1988). Mercury and tetracycline resistance genes and flanking repeats associated with methicillin resistance on the chromosome of Staphylococcus aureus. Molecular Microbiology 2, 289-298.

Skurray, R. A., Rouch, D. A., Lyon, B. R., Gillespie, M. T., Tennent, J. M., Byrne, M. E., Messerotti, L. J. \& May, J. W. (1988). Multiresistant $S$. aureus: genetics and evolution of epidemic Australian strains. Journal of Antimicrobial Chemotherapy 21 (suppl. C), 19-38.

ThaKker-VARIa, S., JensSen, W. D., Moon-McDermott, L., WeinSTEIN, M. P. \& Dubin, D. T. (1987). Molecular epidemiology of macrolides-lincosamides-streptogramin B resistance in Staphylococcus aureus and coagulase-negative staphylococci. Antimicrobial Agents and Chemotherapy 31, 735-743.

Tillotson, L. E., Jenssen, W. D., Moon-McDermott, L. \& Dubin, D. T. (1989). Characterization of a novel insertion of the macrolideslincosamides-streptogramin B resistance transposon Tn554 in methicillin-resistant Staphylococcus aureus and Staphylococcus epidermidis. Antimicrobial Agents \& Chemotherapy 33, 541-550.

Townsend, D. E., GrubB, W. B. \& Ashdown, N. (1983). Genetics of drug resistance in methicillin-resistant Staphylococcus aureus from Australian hospitals. Journal of Hospital Infections 4, 331-337.

Townsend, D. E., Bolton, S., AshDown, N., ANNEAR, D. L. \& GRUBB, W. B. (1986). Conjugative staphylococcal plasmids carrying hitch-hiking transposon similar to Tn554: intracellular and interspecies dissemination of erythromycin resistance. Australian Journal of Experimental Biology and Medical Science 64, 367-379.

Townsend, D. E., Ashdown, N., Bolton, S., Bradley, J. DuckWORTH, E. C., Moorhouse, E. D. \& GrubB, W. B. (1987). The international spread of methicillin-resistant Staphylococcus aureus. Journal of Hospital Infections 9, 60-71.

Ubukata, K., Nonoguchi, R., Matsuhashi, M., Dong Song, M. \& KonNo, M. (1989). Restriction maps of the regions coding for the methicillin and tobramycin resistances on chromosomal DNA in methicillin-resistant staphylococci. Antimicrobial Agents and Chemotherapy 33, 1624-1626.

Weisblum, B. \& Demohn, V. (1969). Erythromycin-inducible resistance in Staphylococcus aureus: survey of antibiotic classes involved. Journal of Bacteriology 98, 447-452. 